\title{
The Application of Big Data Analysis in the Optimization of Mobile Communication Network
}

\author{
Ding Chen ${ }^{1, a}$ \\ ${ }^{1}$ Institute of Technology, East China Jiao Tong University, Nanchang, Jiangxi, China, 330000 \\ a278835001@qq.com
}

\begin{abstract}
At this stage, big data technology has become one of the most practical and widely used technologies, and the application of big data in people's daily life is also very common, and the application of big data technology has brought a lot of convenience to people's lives in society, but at the same time, big data technology has some negative effects on people's lives, information technology often has two sides, and as people's demand for big data technology is increasing, we must make more efforts to promote the development of big data technology. For example, big data technology is very important in mobile communication network optimization Therefore, people are constantly improving the quality of network optimization, and this paper will focus on the application of big data analysis in mobile communication network optimization research and discussion.
\end{abstract}

Keywords: big data analysis; mobile communication network; optimization; thinking

\section{大数据分析在移动通信网络优化中的应用思考}

\author{
陈鼎 1 ,
}

${ }^{1}$ 华东交通大学理工学院，南昌，江西，中国，330000

a278835001@qq.com

\section{摘要}

就现阶段而言，大数据技术成为了当前非常实用并且适用范围非常广泛的技术之一，而在人们的日常 生活中大数据应用的也非常普遍，而大数据技术的应用也给社会给人们的生活带来了很多的便利, 但 是与此同时，大数据技术也给人们生活带来了一些负面影响，信息技术往往是存在两面性的，而随着 人们对大数据技术的要求越来越高, 我们也必须为了推动大数据技术的发展而做出更多的努力。例如 大数据技术在移动移动通信网络优化中是非常重要的, 因此人们也在不断提升网络优化工作的质量, 而本文就将围绕大数据分析在移动通信网络优化中的应用进行研究和探讨。

关键词: 大数据分析; 移动通信网络; 优化; 思考

\section{1. 大数据的技术分析}

大数据技术是时代和科技发展的产物。目前, 在权威领域没有更准确的官方技术解释, 各行各业 对大数据的定义也存在较大差异。一般来说, 大数 据技术就是通过技术的应用来处理海量的数据。在 这些海量数据的基础上, 对这些数据进行专业的处 理、深入的挖掘与分析, 使各种资源得到更合理的 运用。随着大数据技术的不断发展, 其技术已取得 了较大的发展, 越来越多的应用于社会各生产领 域, 成为影响世界发展的关键技术, 是传统技术无 法比拟的, 其技术应用产品可以为高层企业的决策
提供很好的参考, 在基本分析中, 也可以发挥很大 的作用。

\section{2. 移动通信网络概述}

移动通信网络是一种非常好的通信传输媒介, 通过网络的应用, 可以有效地实现客户与客户之间 的通信, 客户与服务器之间的通信。在实现整个通 信过程中，需要强大的专业技术支持。随着我国各 方面的多元化发展, 社会生产与生活对移动通信的 需求越来越高, 对通信质量的要求也越来越高, 这 就对网络运行效果提出了更高的要求。为了保证网 络运行的质量, 需要根据网络运行中存在的问题及 
时优化网络, 以保证网络通信的质量, 大数据分析 技术在网络通信中的应用可以在网络优化中起到 很好的作用。然而, 该技术不仅给网络通信带来了 更好的优化效果,而且对网络通信的进一步发展产 生了很大的影响。

\section{3. 大数据分析技术在移动通信网络优化应用 中存在的问题}

表 1 大数据分析技术在移动通信网络优化应用中存 在的问题

\begin{tabular}{|c|c|c|c|}
\hline $\begin{array}{c}\text { 存在着不 } \\
\text { 少安全风 } \\
\text { 险 }\end{array}$ & $\begin{array}{l}\text { 移动数据 } \\
\text { 存在的问 } \\
\text { 题 }\end{array}$ & $\begin{array}{l}\text { 存在的资 } \\
\text { 金问题 }\end{array}$ & $\begin{array}{l}\text { 用户业务 } \\
\text { 的多元化 }\end{array}$ \\
\hline 目前最大 & 由于大数 & 在具体的 & 在每个区 \\
\hline 的问 题 是 & 据分析技 & 实施过程 & 域的信号 \\
\hline 大数据 分 & 术当前还 & 中, 要综合 & 覆盖区域, \\
\hline 析技术的 & 不够成熟， & 考虑不同 & 将为每个 \\
\hline 应用还不 & 因此在面 & 地 区 数 据 & 用户提供 \\
\hline 够稳定, 因 & 对 海 量 数 & 结构的差 & 多样化的 \\
\hline 此在运用 & 据 时很容 & 异, 移动通 & 服务, 如使 \\
\hline 大数 据 技 & 易出现错 & 信网络建 & 用移动设 \\
\hline 术时, 会有 & 误，并且， & 设成本必 & 备终端观 \\
\hline 很多问题 & 处理 数 据 & 然会增加, & 看视频、打 \\
\hline 不断的显 & 不够及时， & 导致资金 & 电话、汶览 \\
\hline 现, 而这些 & 对于运营 & 不足, 没有 & 新闻或网 \\
\hline 问题都会 & 商而言, 都 & 足够的资 & 页等, 使各 \\
\hline 给网络通 & 是 非常 严 & 金支持, 优 & 项业务得 \\
\hline 信安全造 & 重的影响。 & 化移动通 & 以正常运 \\
\hline 成负面影 & 如 果 不解 & 信网络是 & 行, 特别是 \\
\hline 响, 甚至有 & 决这些问 & 不能实现 & 在用户业 \\
\hline 可能导致 & 题, 那么大 & 的。 & 务密集的 \\
\hline 网 络数据 & 数据分析 & & 地区, 会出 \\
\hline 丢失, 而网 & 技术给移 & & 现网络资 \\
\hline 络 数 据 丢 & 动网络通 & & 源的竞争 \\
\hline 失对于用 & 信所带来 & & 导致网 络 \\
\hline 户而言是 & 的风险 要 & & 拥堵, 一些 \\
\hline 非 常大的 & 大于便利, & & 高流量的 \\
\hline 损失, 并且 & 那 么大数 & & 业务无法 \\
\hline 也会影响 & 据 分析技 & & 正常运行。 \\
\hline 用户对运 & 术在移动 & & \\
\hline 营商的信 & 网络通信 & & \\
\hline & 中的应用 & & \\
\hline & 的价值也 & & \\
\hline & 下降。 & & \\
\hline
\end{tabular}

移动通信网络能够使客户与客户之间的通信, 以及 客户与服务器之间的通信, 都更好得到更好的实现, 但 是想要实现通信, 还需要更加强大的技术支持, 随着人
们对于通信质量的要求越来越高, 对于移动通信工作也 提出了更高的要求, 而为了能够满足人们的需求, 保证 网络的运行质量, 就必须做好网络优化工作, 而网络优 化工作主要根据移动通信网络中所出现的问题进行及 时的解决, 保证网络通信质量, 而在网络通信中应用大 数据分析技术, 就能够有效的做到这一点。但是大数据 分析技术在网络通信中一定有利也有弊, 从某种程度而 言, 大数据分析技术保障了网络的运行质量, 但是大数 据分析技术也存在着不小的风险。

\section{1 存在着不少安全风险}

在移动通信网络中, 大数据分析技术的作用往往是 体现在对数据的处理和分析方面的, 而在资源与资源的 转换中, 大数据分析技术也有着非常大的价值, 而大数 据分析技术与网络通信的质量是紧密相关的, 如果大数 据技术的应用出现问题，那么网络通信的安全也会存在 风险, 而且目前最大的问题是大数据分析技术的应用还 不够稳定，因此在运用大数据技术时，会有很多问题不 断的显现, 而这些问题都会给网络通信安全造成负面影 响, 甚至有可能导致网络数据丢失, 而网络数据丢失对 于用户而言是非常大的损失, 并且也会影响用户对运营 商的信任。因此, 想要更好的在网络通信中应用大数据 分析技术, 保障大数据分析技术的安全应用是非常重要 的。

\section{2 移动数据存在的问题}

随着移动通信网络的覆盖面越来越广, 而通过移动 通信网络所产生的数据资源的数量也在不断增加, 虽然 大数据分析技术本身就是基于大数据的基础之上而建 立的, 但是, 由于大数据分析技术当前还不够成熟, 因 此在面对海量数据时很容易出现错误, 并且, 处理数据 不够及时, 对于运营商而言, 都是非常严重的影响。如 果不解决这些问题, 那么大数据分析技术给移动网络通 信所带来的风险要大于便利, 那么大数据分析技术在移 动网络通信中的应用的价值也会大幅度下降。而为了通 过大数据技术提升移动通信网络的质量和效率, 但是却 不因为大数据分析技术的应用而影响到移动通信网络 的应用, 就还需要更加进一步的探索解决这些问题的对 策。

\section{3 存在的资金问题}

在优化移动通信网络的过程中, 另一个问题是缺乏 资金。基于大数据分析的移动通信网络优化不是一蹴而 就的, 需要经过复杂的系统数据分析过程。在具体的实 施过程中, 要综合考虑不同地区数据结构的差异, 移动 通信网络建设成本必然会增加，导致资金不足，没有足 够的资金支持，优化移动通信网络是不能实现的。 


\section{4 用户业务的多元化}

在每个区域的信号覆盖区域, 将为每个用户提供多 样化的服务, 如使用移动设备终端观看视频、打电话、 汶览新闻或网页等, 使各项业务得以正常运行, 特别是 在用户业务密集的地区, 会出现网络资源的竞争导致网 络拥堵, 一些高流量的业务无法正常运行。对于同一时 间点出现的多元化业务模式, 会让网络数据碎片化并分 散开来，使数据整理不方便。

\section{4. 有效的问题解决对策}

表 2 有效的问题解决对策

\begin{tabular}{|c|c|c|}
\hline $\begin{array}{c}\text { 更好地对存储功 } \\
\text { 能进行利用 }\end{array}$ & $\begin{array}{l}\text { 不断建立和健全 } \\
\text { 相关的管理体系 }\end{array}$ & $\begin{array}{l}\text { 将大数据分析技 } \\
\text { 术应用到位置信 } \\
\text { 息的管理过程中 }\end{array}$ \\
\hline 当移动通信网络 & 大数据技术在人 & 利用大数据技术 \\
\hline 需要对用户的数 & 们的生产和生活 & 建设合理基站, \\
\hline 据进行处理时， & 中, 也逐渐暴露 & 在传统的通信过 \\
\hline 就可以利用大数 & 出更多的问题， & 程中, 由于用户 \\
\hline 据技术的储存功 & 但是人们不应该 & 位置信息难以管 \\
\hline 能, 并且大数据 & 逃避这些问题， & 理, 因此给移动 \\
\hline 技术还能够将数 & 而是应该对这些 & 通信的基站建设 \\
\hline 据虚拟化, 那么 & 问题进行分析并 & 带来了非常大的 \\
\hline 就能够更有利于 & 且予以解决, 完 & 影响, 而通过大 \\
\hline 移动通信网络的 & 善其中的管理体 & 数据技术就能够 \\
\hline 数据处理, 并且 & 系, 让大数据分 & 更好的化解这种 \\
\hline 可以在一定程度 & 析技术的应用能 & 影响, 并且, 更 \\
\hline 上降低移动通信 & 够更加规范。在 & 好的规划位置信 \\
\hline 网络的运行成 & 保证大数据技术 & 息管理。在建立 \\
\hline 本, 使移动通信 & 的应用价值能够 & 基站之后, 发挥 \\
\hline 网络能够更加高 & 全部发挥出来的 & 基站的作用, 避 \\
\hline 效的解决其中所 & 同时, 也要尽可 & 免出现基站混乱 \\
\hline 存在的问题。 & $\begin{array}{l}\text { 能地减少大数据 } \\
\text { 技术可能带来的 } \\
\text { 负面影响 }\end{array}$ & 的情况。 \\
\hline
\end{tabular}

\section{1 更好地对存储功能进行利用}

在移动通信网络中进行大数据分析技术的应用其 实并不全面, 移动通信网络还应该注重对大数据技术的 储存功能的应用, 因为大数据技术能够储存更多的海量 数据, 并且能够对这些数据进行有效的管理, 那么当移 动通信网络需要对用户的数据进行处理时, 就可以利用 大数据技术的储存功能, 并且大数据技术还能够将数据 虚拟化, 那么就能够更有利于移动通信网络的数据处 理, 并且可以在一定程度上降低移动通信网络的运行成 本, 使移动通信网络能够更加高效的解决其中所存在的 问题。

\section{2 不断建立和健全相关的管理体系}

移动通信网络的应用中，大数据分析技术的作用在 不断的显现，但是人们也必须重视其中所存在的网络安 全风险问题, 因为这些网络安全风险问题一旦爆发会给 人们带来非常严重的影响。而且大数据技术在人们的生 产和生活中, 也逐渐暴露出更多的问题, 但是人们不应 该逃避这些问题，而是应该对这些问题进行分析并且予 以解决, 完善其中的管理体系, 让大数据分析技术的应 用能够更加规范。在保证大数据技术的应用价值能够全 部发挥出来的同时, 也要尽可能地减少大数据技术可能 带来的负面影响, 而除此之外, 还应该保障移动数据的 安全性, 完善管理体系, 检测其中所存在的安全风险因 素, 并且保证在出现问题时能够及时的补救, 使每一个 移动通信网络用户都能够安心的使用移动通信网络。

\section{3 将大数据分析技术应用到位置信息的管 理过程中}

用户位置信息管理在优化移动通信网络的过程中 一直是重难点, 而为了能够更好的满足用户的需求, 并 且明确用户位置信息管理, 就应该利用大数据技术建设 合理基站，在传统的通信过程中，由于用户位置信息难 以管理，因此给移动通信的基站建设带来了非常大的影 响, 而通过大数据技术就能够更好的化解这种影响, 并 且, 更好的规划位置信息管理。在建立基站之后, 发挥 基站的作用，避免出现基站混乱的情况。

\section{4 非结构化档案资源数据库的构建}

在移动通信网络信息的应用中，常见的存储方式为 非结构化, 这种存储方式通常用于存储海量信息, 由于 移动通信网络信息的类型比较复杂, 而且随着移动通信 网络应用范围的扩大, 移动数据的数量也会增加。因此, 有必要优化数据库管理, 应用新型数据库管理技术, 进 一步扩展非结构化数据的存储, 运用于移动通信网络中 大数据的管理与存储。

\section{5 分布式计算平台技术的运用}

分布式计算平台为计算机系统提供了一个并行平 台, 解决了信息加载速度慢的问题。在 $4 \mathrm{G}$ 通信时代, 处理海量数据是不可避免的, 分布式计算平台作为云计 算的一种形式，在我国已经得到应用，但在技术上还存 在一些漏洞。将大数据时期的分布式计算平台应用到通 信网络的数据计算中, 可以明确小区的通信流程, 实现 对空闲小区的容量缩减处理, 采用并行信道原则, 从而 保证通信资源的合理利用, 也保证了用户的网络需求。 该平台还实现了不同节点的自动部署和并行化, 对移动 通信的效率的提升具有积极意义。 


\section{5. 结语}

当前人们对移动通信网络的要求越来越高, 而为了 能够满足人们的要求, 就必须使用大数据分析技术, 但 是在使用大数据分析技术的同时, 也要避免大数据分析 技术所带来的风险, 并且积极地利用大数据技术优化移 动通信网络, 完善相关的管理制度。

\section{REFERENCES}

[1] Yangpengcheng Bai. Application Analysis of Big Data in Communication Network Planning[J]. China New Communications. 2019(24)

[2] Daiyun,Xuwei. Research on Optimization of Mobile Communication Network Based on Big Data and Improvement Measures[J]. Science and technology innovation and application. 2019(35)

[3] Wujiang,Huiqin Kang. Explore the Application of Big Data Analysis in Communication Network Optimization[J]. China New Communications. 2019(23)

[4] Kaiyun Guan. Key Technologies of Big Data Processing in Mobile Communication Networks[J].
Electronic Technology and Software Engineering. 2016(19)

[5] Hanxu.5G Key Technologies of Mobile Communication Network[J]. Satellite television and broadband multimedia. 2019(21)

[6] Guojing.Analysis of Traffic Communication Network Based on Big Data Environment[J]. World of Communications. 2017(16)

[7] Yangbin, Ningning Xu. Research on big data analysis technology in mobile communication network optimization[J]. Electronic world. 2020(06)

[8] Hailong Xi. Application of big data analysis technology in mobile communication network optimization[J]. China New Communications. 2019(24)

[9] Gaojin. Application of big data analysis to mobile communication network optimization[J]. message communication. 2019(11)

[10] Mingxiao Liu, Juyong wei. Feasibility Analysis of Mobile Communication Network Optimization Based on Big Data[J]. China New Communications. 2019(15) 